Tribhuvan University, Kathmandu, Nepal

\title{
Some Problems on Approximations of Functions (Signals) in Matrix Summability of Legendre Series
}

\author{
Suresh Kumar Sahani ${ }^{1}$, Vishnu Narayan Mishra ${ }^{2}$ \& Narayan Prasad Pahari ${ }^{3}$ \\ ${ }^{1}$ Department of Mathematics, MIT Campus, Tribhuvan University, Janakpur, Nepal \\ ${ }^{2}$ Department of Mathematics, Indira Gandhi National Tribal University, Lalpur, Amarkantak, \\ Madhya Pradesh 484 887, India \\ ${ }^{3}$ School of Mathematical Sciences, Tribhuvan University, Kathmandu, Nepal \\ Email: ${ }^{1}$ sureshkumarsahani35@gmail.com, ${ }^{2}$ vishnunarayanmishra@gmail.com, 3nppahari@gmail.com
}

Abstract: In this paper, we prove a main theorem dealing the matrix summability of Legendre series using non-negative monotonic non-increasing sequences of function. This paper is more general than [9], [12] and [22].

Keywords: Matrix Method, Matrix Summability, Legendre series.

\section{Introduction}

The Legendre series associated with the Lebesgne integral function in the linear interval $[-1,1]$ is defined by

$$
f(x) \sim \sum_{n=0}^{\infty} a_{n} P_{n}(x)
$$

where $a_{n}=\left(\frac{2 n+1}{2}\right) \int_{-1}^{1} f(t) P_{n}(t) d t$

and Legendre polynomials $P_{n}(x)$, are defined by following expression

$\frac{1}{\sqrt{1-2 x z+z^{2}}}=\sum_{n=0}^{\infty} z^{n} P_{n}(x)$.

However, if the coefficient $a_{n}^{\prime}$ s are not restricted by our relation (2), the series (1) is known as series of Legendre polynomials (see [1], [2], [3], [6], [14], [20], [22], [23]). In 1965, Saxena [19] for the first mathematician who introduced the actual concept of uniform Nörlund Summability of Fourier series and which is defined as follows :

Let $u_{0}(x)+u_{1}(x)+u_{2}(x)+\cdots+u_{n}(x)+\cdots$

be any infinite series and define

$V_{m}(x)=u_{0}(x)+u_{1}(x)+u_{2}(x)+\cdots+u_{m}(x)$ 
Let $\left\{P_{n}\right\}$ be a sequence of constants, real or complex, and let us write

$P_{n}=p_{0}+p_{1}+p_{2}+\cdots+p_{n}$

If their exits a function $V=v(x)$

such that $\frac{1}{P_{n}} \sum_{k=0}^{n} p_{k}\left\{U_{n-k}(x)-U(x)\right\}=\mathrm{o}(1)$

uniformly in a set $\mathrm{E}$ in which $U(x)$ is bounded, then the series (4) is summable $\left(N, p_{n}\right)$ uniformly in $\mathrm{E}$ to the sum $U$. Sahani et al. ([16], [17]), Mishra et al.([9], [1], [10], [13], [12]), and Prasad [15], the first Mathematicians to use a study on the behavior of absolute permanent matrix transformation, on the degree of approximation of a function by Nörlund means of its Fourier Laguere series, trigonometric approximation of functions $f(x, y)$ of generalized Lipschitz class by double Hausdorff matrix summability method, trigonometric approximation $L_{p}$-norm. On the degree approximation of signals belonging to generalized weighted Lipschitz $W^{\prime}\left(L^{r}, \xi(t)\right)(r \geq 1)$ Class- by matrix $\left(C^{\prime}, N_{p}\right)$ operator of conjugate series, trigonometric approximation of periodic signals belonging to generalized weighted Lipschitz $W^{\prime}\left(L_{r}, \xi(t)\right),(r \geq 1)$ - class by Nörlund- Euler $\left(N, p_{n}\right),(E, q)$ operation of conjugate series of Fourier series, Using linear operator to approximate signals of $\operatorname{Lip}(\alpha, p),(p \geq 1)$ class and on the Nörlund summability of Legendre series. In an attempt to make an advance study in this direction we, in this paper establish a more general result than those of [8], [21], and [15] so their results come out as particular case.

\section{Definition:}

Let $T=\left(a_{n, k}\right)$ be an infinite triangular matrix of real constants and $t_{n}(x)$ denote the T-transform of $\left\{S_{n}\right\}$.

Then

$$
\begin{aligned}
t_{m}(x) & =\sum_{k=0}^{m} a_{m, k}\left(S_{k}(x)-S(x)\right) \\
& =\sum_{k=0}^{m} a_{m, m-k}\left(S_{m-k}(x)-S(x)\right) .
\end{aligned}
$$

If their exits a function $S(x)$ such that $t_{m}(x)=0(1)$, as $m \rightarrow \infty$

uniformly in $\mathrm{E}$ in which $S(x)$ is bounded, then the infinite series $\sum u_{n}$ is summable $(T)$ uniformly in $\mathrm{E}$ to $S(x)$ (see [9]).

\section{Main Results}

\section{Theorem:}

If $\left\{a_{n, k}\right\}_{k=0}^{\infty}$ be a real, non-negative monotonic non-increasing sequence and $T=\left(a_{n, k}\right)$ be an infinite triangular matrix, where $A_{n, v}=\sum_{k=0}^{v} a_{n, n-k}, A_{n, n}=1$ for $n \geq 0$ and if

$\Psi(t)=\int_{0}^{t}|\Psi(u)| d u=O\left(\frac{\mathrm{x}\left(\frac{1}{t}\right) \cdot t}{\log t^{-1}}\right)$

as $t \rightarrow+0$, uniformly in $\mathrm{E}$ in $-1<x<1$ in which $f(x)$ is bounded, where $\mathrm{X}\left(\frac{1}{t}\right)$ and $\frac{\mathrm{X}\left(\frac{1}{t}\right) \cdot t}{\log t^{-1}}$ increases monotonic with $t$, then the Legendre series (1) is summable $(T)$ uniformly in E to the sum $f(x)$.

For the proof of our theorem, we require the following 
Lemma 1 (see[21])

$\sum_{v=0}^{n}(2 v+1) P_{v}(x) P_{v}(y)=\frac{(n+1)\left[P_{n+1}(y) P_{n}(x)-P_{n}(y) . P_{n+1}(x)\right]}{y-x}$.

This identity is known as Christoffel's formula of summation.

\section{Lemma 2 (see [4])}

For $0<\varepsilon \leq \gamma \leq \pi-\varepsilon$,

$P_{n}(\cos \gamma)=\sqrt{\frac{2}{\pi n \sin \gamma}} \cos \left[(n+1) \gamma-\frac{\pi}{4}\right]+\mathrm{O}\left(n^{\frac{-3}{2}}\right)$.

\section{Lemma 3 (see [7])}

If $\left\{a_{n, k}\right\}$ is a non-negative and non-increasing sequence with respect to $\mathrm{k}$, then for $0 \leq a<b<\infty$, $0 \leq t \leq \pi$ and for every $n$, we have

$$
\left|\sum_{k=a}^{b} a_{n, k} e^{i(n-k) t}\right|=\mathrm{O}\left[\frac{1}{t} \cdot a_{n, n-\tau}\right]
$$

Where $\tau$ is the integral part of $\frac{1}{t}$.

\section{Lemma 4:}

For $0 \leq t<\frac{1}{n},\left|N_{n}(t)\right|=0(n)$ as $n \rightarrow \infty$.

Proof:

We know that $N_{n}(t)=\sum_{k=0}^{n} a_{n, n-k} \frac{\sin (n-k+1) t}{\sin \frac{t}{2}}$

$$
\begin{aligned}
\therefore\left|N_{n}(t)\right| & \leq\left|\sum_{k=0}^{n} a_{n, n-k} \frac{\sin (n-k+1) t}{\sin \frac{t}{2}}\right| \leq\left|\sum_{k=0}^{n} a_{n, n-k} \frac{(2 n-2 k+2) \sin \frac{t}{2}}{\sin \frac{t}{2}}\right| \leq 4 n \sum_{k=0}^{n} a_{n, n-k} \\
& =4 n \mathrm{O}(1),(\text { by hypothesis of theorem }) \\
& =\mathrm{O}(1) .
\end{aligned}
$$

\section{Lemma 5 :}

$$
\text { For } \frac{1}{n} \leq t \leq \pi, N_{n}(t)=O\left(\frac{A_{n, v}}{t}\right), v \leq n
$$

\section{Proof :}

$$
\begin{aligned}
\therefore\left|N_{n}(t)\right| & \leq\left|\sum_{k=0}^{n} a_{n, n-k} \frac{\sin (n-k+1) t}{\sin \frac{t}{2}}\right| \leq\left|\frac{1}{\sin \frac{t}{2}} \operatorname{Im} \sum_{k=0}^{n} a_{n, n-k} e^{i(n-k+1) t}\right| \\
& \leq \frac{\pi}{t} \operatorname{Im} \sum_{k=0}^{n} a_{n, n-k} e^{i(n-k) t} \leq \frac{\pi}{t}\left|\sum_{k=0}^{n} a_{n, n-k} e^{i(n-k) t}\right| \\
& =\frac{\pi}{t} A_{n, v} \quad(\text { by, lemma 3) } \\
& =\mathrm{O}\left(\frac{A_{n, v}}{t}\right) .
\end{aligned}
$$




\section{Proof of the theorem.}

The $n^{\text {th }}$ partial sum of the series (1) is given by

$$
\begin{aligned}
S_{n}(x) & =\sum_{v=0}^{n} a_{v} p_{v}(x) \\
& =\sum_{v=0}^{n}\left(v+\frac{1}{2}\right) \int_{-1}^{1} f(y) p_{v}(y) p_{v}(x) d y \quad(\text { by } 2) \\
& =\sum_{v=0}^{n}\left(\frac{2 v+1}{2}\right) \int_{-1}^{1} f(y) p_{v}(y) p_{v}(x) d y \\
& =\left(\frac{n+1}{2}\right) \int_{-1}^{1}\left\{\frac{p_{n+1}(y) p_{n}(x)-p_{n+1}(x) p_{n}(y)}{y-x}, f(y) d y\right\}
\end{aligned}
$$

Putting $f(y)=1$, then

$\mathrm{I}=\left(\frac{n+1}{2}\right) \int_{-1}^{1} \frac{p_{n+1}(y) p_{n}(x)-p_{n+1}(x) p_{n}(y)}{y-x} d y$

Thus, $S_{n}(x)-f(x)=\frac{n+1}{2} \int_{-1}^{1}[f(y)-f(x)] \cdot \frac{P_{n+1}(y) \cdot P_{n}(x)-P_{n+1}(x) \cdot P_{n}(y)}{y-x} d y$

Hence by (8), we have

$$
\begin{aligned}
t_{n}(x) & =\sum_{k=0}^{n} a_{n, n-k}\left\{s_{n-k}(x)-f(x)\right\} \\
& =\sum_{k=0}^{n} a_{n, n-k} \frac{n-k+1}{2} \int_{-1}^{n} \frac{n-k+1}{2}\{f(y)-f(x)\} \frac{\left\{P_{n-k+1}(y) P_{n-k}(x)-P_{n-k}(y) \cdot P_{n-k+1}(x)\right\}}{y-x} d y \\
& =\sum_{k=0}^{n-1}\left(\frac{n-1+k}{2} a_{n, n-k}\right) \int_{-1}^{1}[f(y)-f(x)]\left\{\frac{\left\{P_{n-k+1}(y) P_{n-k}(x)-P_{n-k}(y) \cdot P_{n-k+1}(x)\right\}}{y-x} d y\right\}+O(1)
\end{aligned}
$$

We define a positive number $\mathrm{S}$ which is less than 1 i.e. $S<1$ and also consider it as the sum of other two positive numbers $\beta$ and $\alpha$.

Let $\delta>0$ and $0<\delta<\gamma$ and $\beta x, \beta x^{1}$ be two continuous functions of $\mathrm{x}$ such that $\beta x, \beta x^{1} \epsilon(-1,1)$ and which lies within $\delta \leq \beta x \leq \beta, \delta \leq \beta x^{\prime} \leq \beta$.

Thus, for $-1+S \leq x \leq 1-S$, we have,

$$
\begin{aligned}
t_{n}(x)= & \sum_{k=0}^{n-1} a_{n, n-k}\left(\frac{n-k+1}{2}\right)\left[\int_{-1}^{x-\beta x}+\int_{x-\beta x}^{x+\beta x^{1}}+\int_{x+\beta x^{1}}^{1}\right] \\
& \int_{-1}^{1}[f(y)-f(x)] \frac{\left[P_{n-k+1}(y) P_{n-k}(x)-. P_{n-k}(y) \cdot P_{n-k+1}(x)\right]}{y-x} d y+O(1) \\
= & \sum_{k=0}^{n-1} a_{n, n-k}\left[A_{n-k}(x)+B_{n-k}(x)+C_{n-k}(x)\right]+\mathrm{O}(1) .
\end{aligned}
$$

Hobson [8] has shown that uniformly for $s-1 \leq x \leq 1-s$,

$$
\lim _{n \rightarrow \infty} A_{n-k}(x)=0 \text {, }
$$

and $\quad \lim _{n \rightarrow \infty} C_{n-k}(x)=0$.

Now let us suppose that $x=\cos \theta, y=\cos \phi, 0<\theta<\pi$,

$0<\phi<\pi, 0<\varphi<\pi, 1-\gamma=\cos \rho$ 


$$
\begin{aligned}
& 1-(\beta+\gamma)=1-s=\cos (\rho+\sigma), \\
& \rho>0, \sigma>0, \text { then we have } \\
& 0<\rho<\frac{\pi}{2} \text { and } \rho+\sigma<\frac{\pi}{2} .
\end{aligned}
$$

Thus if $\xi$ denote the minimum of $\left[\cos ^{-1} u-\cos ^{-1}(u+\beta)\right]$ for $u \in(1,1-\beta)$,

we have on the lines of Sansone [21],

$B_{n-k}(\cos \theta)=\frac{n-k+1}{2} \int_{\theta-\xi}^{\theta+\xi}[f(\cos \phi)-f(\cos \theta)] \operatorname{Sin} \phi \cdot \frac{p_{n-k+1}(\cos \phi) p_{n-k}(\cos \theta)-p_{n-k}(\cos \phi) p_{n-k+1}(\cos \theta)}{\cos \phi-\cos \theta} d \phi$.

In which $\rho+\sigma \leq \theta \leq \pi-(\rho+\sigma), 0<\xi \leq \sigma$, using lemma 2

and for $\alpha=\beta=0$ we get after simplification

$$
B_{n-k}(\cos \theta)=\frac{1}{2 \pi \sqrt{\sin \theta}} \int_{\theta-\xi}^{\theta+\xi}\{f(\cos \phi)-f(\cos v) \sqrt{\sin \phi} \cdot\}\left\{\frac{\sin (n-k+1)(\theta-\phi)}{\sin \frac{\theta-\phi}{2}}+\frac{\sin (n-k+1)\left(\theta+\phi-\frac{\pi}{2}\right)}{\sin \frac{\theta-\phi}{2}}+O\left[\frac{1}{(n-k)^{2}}\right]\right\} d \varphi
$$

Thus,

$$
\begin{aligned}
t_{n}(x) & =\frac{1}{2 \pi \sqrt{\sin \theta}} \sum_{k=0}^{n-1} a_{n, n-k} \int_{\theta-\xi}^{\theta+\xi}[f(\cos \phi)-f(\cos \theta)] \sqrt{\sin \phi} \cdot\left\{\frac{\sin (n-k+1)(\theta-\phi)}{\sin \left(\frac{\theta-\varphi}{2}\right)}+\frac{\sin (n-k+1)\left(\theta+\phi-\frac{\pi}{2}\right)}{\sin \left(\frac{\theta+\varphi}{2}\right)}\right\}+\mathrm{O}\left[\frac{1}{(n-k)^{2}}\right] d \varphi \\
& =J_{1}+J_{2}+J_{3}
\end{aligned}
$$

\section{For $J_{1}$,}

$$
\begin{aligned}
J_{1}= & \frac{1}{\pi \sqrt{\sin \theta}} \sum_{k=0}^{n-1} a_{n, n-k} \int_{0}^{\xi}[f(\cos (\theta-t))-f(\cos \theta)] \sqrt{\operatorname{Sin}(\theta-t)} \cdot \frac{\sin (n-k+t) t}{\sin \frac{t}{2}} d t \\
= & \frac{1}{\pi \sqrt{\sin \theta}} \int_{0}^{\xi}[f\{\cos (\theta-t)\}-f(\cos \theta)] \sqrt{\sin (\theta-t)} \cdot \sum_{k=0}^{n-1} a_{n, n-k} \frac{\sin (n-k+1) t}{\sin \frac{t}{2}} d t \\
& =\mathrm{O}\left[\int_{0}^{\xi}|\Psi(t)| \cdot N_{n}(t) d t\right] \quad \text { (by given condition) } \\
& =\mathrm{O}\left[\int_{0}^{\frac{1}{n}}+\int_{\frac{1}{n}}^{\xi}\right]|\Psi(t)|\left|N_{n}(t)\right| d t \\
& =J_{1.1}+J_{1.2}
\end{aligned}
$$

Using lemma 4 in $J_{1.1}$, we have

$$
\begin{aligned}
J_{1.1} & =\mathrm{O}\left[\int_{o}^{\frac{1}{n}}|\Psi(t)|\left|N_{n}(t)\right| d t\right] \\
& =\mathrm{O}\left[n \int_{0}^{\frac{1}{n}}|\Psi(t)| d t\right]=\mathrm{O}\left[n O\left(\frac{\frac{1}{n} \mathrm{X}(n)}{\log n}\right)\right], \text { by given condition } \\
& =\mathrm{O}\left[\left(\frac{\Psi(n)}{\log n}\right)\right] \\
& =\mathrm{O}(1) \text { as } n \rightarrow \infty
\end{aligned}
$$


$=\mathrm{O}(1)$, as $n \rightarrow \infty$, uniformly in $E$.

Again, using lemma 5, we have

$$
\begin{aligned}
& J_{1.2}=\mathrm{O}\left[\int_{\frac{1}{n}}^{\xi}|\Psi(t)|\left|N_{n}(t)\right| d t\right] \\
& =\mathrm{O}\left[\int_{\frac{1}{n}}^{\xi}|\Psi(t)| \frac{A_{n, v}}{t} d t\right] \\
& =O\left[\frac{A_{n, v}}{t} \Psi(t)\right]_{\frac{1}{n}}^{\xi}+\mathrm{O}\left[\int_{\frac{1}{n}}^{\xi} \frac{A_{n, v}}{t^{2}} \Psi(t) d t\right]+\mathrm{O}\left[\int_{\frac{1}{n}}^{\xi} \frac{\Psi(t)}{t} d\left(A_{n, v}\right)\right] \\
& =\mathrm{O}\left[\frac{A_{n, v}}{t} O\left(\frac{t \cdot \mathrm{X}\left(\frac{1}{t}\right)}{\log t^{-1}}\right)\right]_{\frac{1}{n}}^{\xi}+\mathrm{O}\left[\int_{\frac{1}{n}}^{\xi} \frac{A_{n, v}}{t^{2}} O\left(\frac{t \cdot \mathrm{X}\left(\frac{1}{t}\right)}{\log t^{-1}}\right)\right]+\mathrm{O}\left[\int_{\frac{1}{n}}^{\xi} O\left(\frac{t \cdot \mathrm{X}\left(\frac{1}{t}\right)}{\log t^{-1}}\right) d \frac{A_{n}, v}{t}\right] \\
& =\mathrm{O}\left[A_{n, \frac{1}{\xi}} \frac{\mathrm{x}\left(\frac{1}{\xi}\right)}{\log \left(\frac{1}{\xi}\right)}\right]+\mathrm{O}\left[\frac{A_{n, n} \mathrm{X}(n)}{\log n}\right]+\mathrm{O}\left[\frac{\mathrm{x}(n)}{n \log n} \int_{\frac{1}{n}}^{\xi} \frac{A_{n, v}}{t^{2}} d t\right]+\mathrm{O}\left[\frac{\mathrm{x}(n)}{n \log n} \int_{\frac{1}{n}}^{\xi} \frac{d\left(A_{n, v}\right)}{t}\right], \text { (by hypothesis of the theorem) } \\
& =\mathrm{O}(1)+\mathrm{O}\left[O(1) \frac{\mathrm{X}(n)}{\log n}\right]+\mathrm{O}\left[\frac{\mathrm{X}(n)}{n \log n}\left\{\left(\frac{A_{n, v}}{t}\right)_{\frac{1}{n}}^{\xi}+\int_{\frac{1}{n}}^{\xi} \frac{d\left(A_{n, v}\right)}{t}\right\}\right]+\mathrm{O}\left[\frac{\mathrm{X}(n)}{n \log n} \int_{\frac{1}{n}}^{\xi} \frac{d\left(A_{n, v}\right)}{t}\right] \\
& =\mathrm{O}(1)+\mathrm{O}\left[\frac{\mathrm{X}(n)}{\log n}\right]+O\left[\frac{\mathrm{X}(n)}{n \log n}\left(\frac{A_{n}, v}{t}\right)_{\frac{1}{n}}^{\xi}\right]+\mathrm{O}\left[\frac{\mathrm{X}(n)}{n \cdot \log n} \int_{\frac{1}{n}}^{\xi} \frac{d\left(A_{n, v}\right)}{t}\right] \\
& =\mathrm{O}(1)+\mathrm{O}\left[\frac{\mathrm{X}(n)}{\log n}\right]+\mathrm{O}\left[\frac{\mathrm{X}(n)}{\log n} A_{n, n}\right]+\mathrm{O}\left[\frac{\mathrm{X}(n)}{n \log n} \int_{\frac{1}{\xi}}^{n} u d\left(A_{n, u}\right)\right]+\mathrm{O}\left[\frac{\mathrm{X}(n)}{n \cdot \log n}\left(\frac{{ }_{n, \frac{1}{\xi}}}{\xi}\right)\right]\left(\because u=\frac{1}{t}\right) \\
& =\mathrm{O}(1)+\mathrm{O}\left[\frac{\mathrm{X}(n)}{n \log n} n \int_{\frac{1}{\xi}}^{n} d\left(A_{n, u}\right)\right]+\mathrm{O}(1)+\left[\frac{\mathrm{X}(n)}{\log n} \mathrm{O}(1)\right]+\mathrm{O}\left[\frac{\mathrm{X}(n)}{\log n}\right] \\
& =\mathrm{O}(1)+\left[\frac{\mathrm{X}(n)}{\log n} \sum_{k=0}^{n} a_{n, n-k}\right]+\mathrm{O}(1)+\mathrm{O}\left(\frac{\mathrm{X}(n)}{\log n}\right)+\left(\frac{\mathrm{X}(n)}{\log n}\right) \\
& =\mathrm{O}(1)+\mathrm{O}\left[\frac{\mathrm{X}(n)}{\log n}\right]+\left[\frac{\mathrm{X}(n)}{\log n}\right]+\mathrm{O}(1) \\
& =\mathrm{O}(1)+\mathrm{O}(1)+\mathrm{O}(1)+\mathrm{O}(1) \text {, as } n \rightarrow \infty \text {, (by the given condition ) } \\
& =\mathrm{O}(1) \text {, as } n \rightarrow \infty \text {, uniformly in } E \text {. }
\end{aligned}
$$

Similarly, $J_{2}=O(1)$, as $n \rightarrow \infty$, and $J_{3}=O(1)$, as $n \rightarrow \infty$, uniformly in $E$.

Combining (15), (16),(17), (18) and (19), we get the required results.

This completes the proof of the theorem. 


\section{Conclusion}

In this paper, we prove a general theorem for some problems on approximation of function (signals) using in matrix summability of Legendre series. This general theorem enriches the literature of summability theory and create basis for future researchers.

\section{Acknowledgements}

The authors are thankful to the referees for their constructive suggestions for the improvement of the content of the paper.

\section{References}

[1]. Ali A., Suleiman M. and Soheil S. (2012). An Operational Matrix Based on Legendre polynimials for solving Fuzzy Fractional -Order Diff. Equations. Hindawi Publishing Corporation, Abstract and Applied Analysis. Vol.(2012), Article ID 505903,29 pages.

[2]. Dattoli G, Cesarano C., Martinelli M.R. and Ricci P.E. (2011). A novel theory of Legendre Polynomails. Math Comput. Modeling .54: 80-87.

[3]. Dattoli G. and Ricci P.E. (2001). A note on Legendre polynomials. Int. J. Non-linear Sci. Numer. Simul. 2: 365-370.

[4]. Foa A. (1943). Sulla Sommabilita forte della Serie dilegendre. Bull. Un. Mat. Ital. , 2 (5): 18-27.

[5]. Hobson E.W. (1909). on the representation by a series of Legendre series is functions. Proceeding of London Mathematical Soc. .2 (7): 24-29.

[6]. Khan S. and AL-Gunah A.A. (2012). summation formulae for the Legendre polynomails, Acta Math. Univ. Comenianae. LXXXI. 1 : 127-139.

[7]. Kishore N. and Hota G.C. (1971). On absolute matrix Summability Fourier series. Indian Journal of Mathematics . 2(13): 99-110.

[8]. Lal S. and Pratap A. (1999). On uniform matrix summability of a Fourier series. Bull. Calcutta Math. Society. 91(5): 427-432.

[9]. Mishra A., Mishra V.N. and Mursaeen M. (2020). Trigonometric approximation of functions $f(x, y)$ of generalized Lipschitz class by double Hansdroft matrix summability method Advances in differen equations 2020, Article no. 681.

[10]. Mishra L.N., Mishra V.N., Khatri K. and Deepmala (2014). On the trigonometric approximation of signals belonging to generalized Lipschitz $W^{1}\left(\mathrm{~L}_{\mathrm{r}}, \xi(\mathrm{t})\right)$, $(\mathrm{r} \geq 1)$ class by matrix $\left(C^{1}, \mathrm{~N}_{\mathrm{p}}\right)$ operator of Conjugate series of its Fourier series. Applied Mathematics and Computation. 237: 252-263.

[11]. Mishra V. N. and Mishra L. N. (2012). Trigonometric approximation of functions in Lp-norm. Int. J. of Contemporary Mathematical Sciences.7(19): 909-918. 
[12]. Mishra V. N., Khatri K., and Mishra L.N. (2013). Using linear operators to approximate signals of Lip. $(\alpha, p),(p \geq 1)$ class. Filomat. 27:21353-363.

[13]. Mishra V.N., Khatri K., Mishra L.N. and Deepmala (2014). Trigonometric approximation of periodic signals belonging to generalized weighted Lipschitz $W^{1}\left(\mathrm{~L}_{\mathrm{r}}, \xi(\mathrm{t})\right),(\mathrm{r} \geq 1)$ - class by Norland -Euler $\left(\mathrm{N}, \mathrm{P}_{\mathrm{n}}\right)(\mathrm{E}, \mathrm{a})$ operator at conjugated series of its Fourier series. Journal of Classical Analysis. 5(2): 91-105.

[14]. Khan N.U., Usman T. (2018). Certain generating functions of Hermite- Berniulli - Legendre Polynomials. Ufimskii Mathematicheskii Zhurnal. 10(2): 118-126

[15]. Prasad K. (1979). On the uniform Nörlund summability of Legendre series. Indian J. Pure Appl. Math.10(10): 1298-1302.

[16]. Sahani S.K. and Mishra V.N. (2020). A study on behavior of absolute permanent matrix Transformation. Int. Journal Math. Trends and Technology . 66(12): 122-127.

[17]. Sahani S.K., Mishra V.N. and Pahari N.P. (2020). On the degree of approximation of a function by Nörlund means of its Fourier Laguerre series. Nepal Journal of Mathematical Sci. ,1: 65-70.

[18]. Sansone, G., (1959) Orthogonal function, English Edition.

[19]. Saxena A. (1965). On uniform Nörlund summability of Fourier series. Proc. Natn. Inst. Sci. India. 32: $502-508$.

[20]. Shehata A. (2016). A new kind of Legendre matrix polynomials. Gazi Univ. Journal Sci. GUJ. Sci. 29: 435-457.

[21]. Tripathi L.M. (1985). On uniform triangular matrix summability of Legendre series. Vijnana Parishad Anusandhan Patrika. 28(1).

[22]. Upadhya L. and Shehata (2011). A Legendre matrix polynomails and its applications. Int. Trans. Math. Sci. Computation. 4 : 291-310.

[23]. Wang H and Xiang S.(2012). On the convergence rates of Legengre approximation. Mathematics of Computation. 81(278): 861-877. 